\title{
Akademiker-Erythem
}

\author{
Eine 20-jährige Studentin stellte sich mit einer seit \\ zwei Monaten bestehenden netzartigen Hyperpig- \\ mentierung an der Vorderseite beider Oberschenkel \\ vor (Abb.). Sie war sonst gesund und hatte lediglich in \\ den vergangenen sechs Monaten wegen einer Akne \\ vulgaris Isotretinoin eingenommen.
}

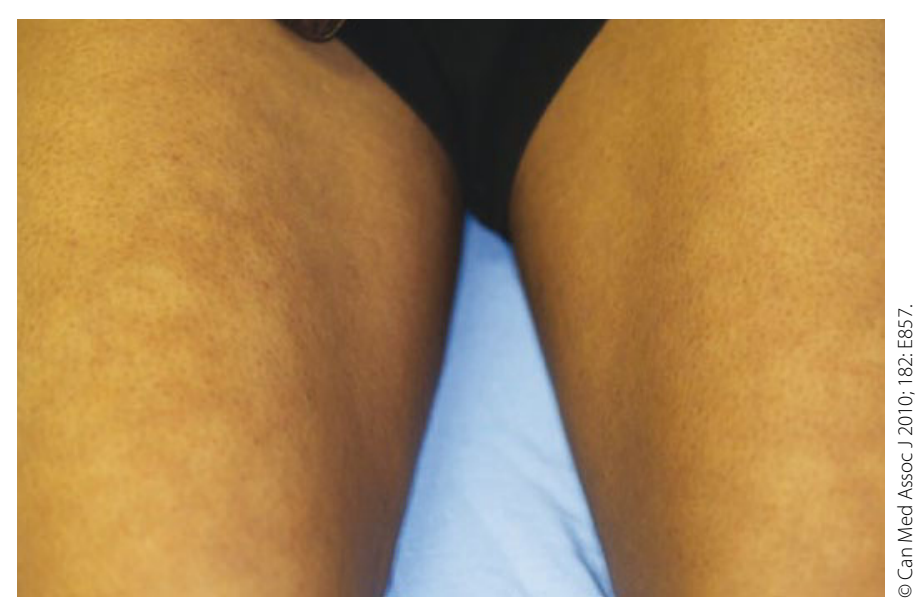

Hyperpigmentierung an den Oberschenkeln.
— Ein Trauma oder ungewöhnliche Gewohnheiten wurden nicht angegeben, an Medikamenten nahm sie nur orale Kontrazeptiva ein. Bei der genaueren Anamnese konnte man jedoch erfahren, dass sie täglich mehrere Stunden lang den auf den Oberschenkeln ruhenden Laptop benutzte. Dieser Umstand sowie der Lokalbefund führten zur Diagnose eines Erythema ab igne. Dabei handelt es sich um eine gutartige Hyperpigmentierung mit retikulärem Muster, die auftritt, wenn die Haut wiederholt mit Hitze in Kontakt kommt. Sie betrifft etwa $3 \%$ der Bevölkerung und tritt in den meisten Fällen beim Gebrauch von Heizkissen und Wärmflaschen auf.
Große Hitze führt zu Schädigungen der Epidermis entlang der Blutgefäße, wodurch es zu netzartig konfigurierten Hämosiderinablagerungen kommt. Die Zeitdauer bis zum Auftreten des Erythema ab igne ist sehr variabel und kann zwischen zwei Wochen und einem Jahr nach Beginn der Hitzeexposition auftreten.

Außer dem Weglassen der Hitzequelle gibt es keine Behandlungsmöglichkeit. Die Hautveränderungen gehen aber in den meisten Fällen innerhalb von Wochen bis Monaten spontan zurück. Bei ausgeprägten Hautschäden wurde die topische Anwendung von 5-Fluorouracil empfohlen.

\section{Macht Diabetes depressiv?}

Es wird immer wieder diskutiert, ob Menschen mit Diabetesrisiko durch die Insulinresistenz häufiger depressiv werden oder ob Depression insulinresistent macht. In beiden Fällen besteht eine Koinzidenz, die letztendlich zur Erhöhung des Diabetesrisikos und zur Manifestation eines Diabetes mellitus führen kann.

- Als Bestandteil der FIND2D-Studie wurden 2712 Teilnehmer in Finnland zu einem Gesundheitscheck eingeladen. Die depressive Stimmungslage wurde mit dem Beck Depression Inventory ermittelt. Alle Personen machten einen oralen Glukosetoleranztest, und die Glukosetoleranzsituation wurde bestimmt.

Depressive Symptome zeigten $14,4 \%$ der Personen mit normaler Glukosetoleranz, 13,7\% der Probanden mit einer ge- störten Glukosetoleranz, 14,8\% der Personen mit neu diagnostiziertem Diabetes und 26,4\% der Patienten mit bestehendem Diabetes. Teilnehmer mit bekanntem Diabetes haben eine signifikant höhere Wahrscheinlichkeit für Depressionen als die Personen mit gestörter Glukosetoleranz oder nicht diagnostiziertem Diabetes. Allerdings egalisierte sich diese Assoziation, nachdem auf Einflussfaktoren adjustiert wurde.

\section{Kommentar}

Die Studie macht es nicht einfacher. Gibt es nun einen Zusammenhang zwischen Diabetes und Depression oder nicht? Dieser populationsbasierte Survey zeigt, dass es einen Zusammenhang zwischen bestehendem Diabetes und Depression gibt, der aber durch viele Einflussfaktoren verwischt oder überdeckt ist. Man könnte das Ergebnis dahingehend interpretieren, dass Diabetes per

\section{Kommentar}

Der Schlüssel zur Diagnose lag in diesem Fall einmal mehr in der Anamnese. Der tägliche stundenlange Umgang mit dem Laptop war für die Studentin sicher selbstverständlich und nicht der Rede wert. Wäre sie nicht gezielt darauf angesprochen worden, so hätte sie diese Angabe möglicherweise nicht gemacht.

H. S. FÜESSL .

- D. Botten el al.

(Dalhousie Health Service, Dalhousie University, Halifax, NS): Academic branding: erythma ab igne and use of laptop computers. Can. Med. Assoc. J. 2010; 182: E857. se nicht depressiv macht, aber die Diagnose eines Diabetes einen Patienten depressiv werden lassen kann. Es stellt sich damit unweigerlich die Frage, ob die Diagnose des Diabetes für den Patienten aufgrund kognitiver Verarbeitungsprozesse ein höheres Depressionsrisiko bedeutet oder ob die zugrunde liegende Insulinresistenz der entscheidende Trigger ist.

Man kann schlussfolgern, dass die Diagnose eines Diabetes den Patienten einem deutlich höheren Risiko aussetzt, depressiv zu werden, und wir das in der Schulung und Intervention bei Diabetespatienten als auch Risikopersonen unbedingt im Fokus haben müssen.

P. E. H. SCHWARZ =

- P. Mantyselka et al.

Association of depressive symptoms with impaired glucose regulation, screen-detected, and previously known type 2 diabetes: findings from the Finnish D2D Survey. Diabetes Care 34 (2011) 71-76 